Supporting Information

(13 pages, including 10 Figures and 1 Table)

\title{
Controllable conversion from single-crystal nanorods to polycrystalline nanosheets of NiCoV-LTH for oxygen evolution reaction at large current density
}

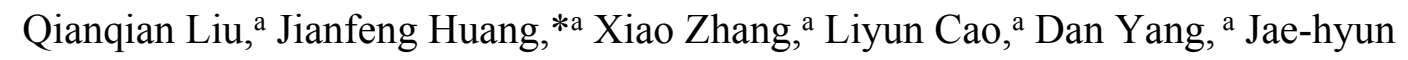
$\mathrm{Kim}^{\mathrm{b}}$ and Liangliang Feng*a

a School of Material Science and Engineering, International S\&T Cooperation Foundation of Shaanxi Province, $\quad$ Xi'an Key Laboratory of Green Manufacture of Ceramic Materials, Shaanxi University of Science and Technology, Weiyang University Campus, Xi'an 710021, China.

${ }^{\mathrm{b}}$ Research Laboratory of Hydrothermal Chemistry, Faculty of Science and Technology, Kochi University 2-5-1, Akebono-cho, Kochi, 780-8520, Japan.

E-mails: huangjf@sust.edu.cn; fengll@sust.edu.cn 


\section{Content}

Experimental Details

SEM images and TEM images. Figure S1

SEM images. Figure S2

SEM images. Figure S3

survey spectra. Figure S4

The atomic percentage. Figure S5

OER polarization curves. Figure S6

The CV curves. Figure S7

ECSA-normalized LSV curves. Figure S8

XRD pattern; Raman spectra; SEM images; TEM image; HRTEM images of PNiCoV-LTH/NF after OER test Figure S9

Overall water-splitting performance curve. Figure S10

Comparison of the electrocatalytic performance of P-NiCoV-LTH/NF with other OER catalysts reported recently in $1 \mathrm{M} \mathrm{KOH}$ solution Table S1

References 


\section{Experimental Details}

\section{Chemicals and Reagents}

Cobalt chloride hexahydrate $\left(\mathrm{CoCl}_{2} \cdot 6 \mathrm{H}_{2} \mathrm{O}\right)$, Vanadium chloride $\left(\mathrm{VCl}_{3}\right)$, hexamethylenetetramine (abbreviation: HMT, $\mathrm{C}_{6} \mathrm{H}_{12} \mathrm{~N}_{4}$ ), $\mathrm{NH}_{4} \mathrm{~F}$, potassium hydroxide $(\mathrm{KOH})$ and ethanol absolute $(\mathrm{CH} 3 \mathrm{CH} 2 \mathrm{OH})$ were purchased from Sinopharm Chemical Reagent Co., Ltd. (China). Ni foam (NF, thickness: $1.5 \mathrm{~mm}$, bulk density: $0.23 \mathrm{~g} / \mathrm{cm}^{3}$; number of pores per inch: 110) was purchased from Suzhou JiaShiDe metal foam Co., Ltd. Ultrapure water $(>18.2 \mathrm{M} \Omega \mathrm{cm})$ was provided by a PALL PURELAB Plus system.

\section{The synthesis of NiCoV-LTH/NF}

$\mathrm{Ni}$ foam was first immersed carefully by $3 \mathrm{M} \mathrm{HCl}$ solution and washed in succession with acetone for 15 min respectively, and then washed thoroughly with ultrapure water and ethanol for three times to insure clean surface. In a typical experiment, $0.4282 \mathrm{~g} \mathrm{CoCl}_{2} \cdot 6 \mathrm{H}_{2} \mathrm{O}, 0.2835 \mathrm{~g} \mathrm{VCl}_{3}, 2.102 \mathrm{~g} \mathrm{HMT}$ and $0.185 \mathrm{~g} \mathrm{NH}_{4} \mathrm{~F}$ were added to $30 \mathrm{~mL}$ of ultrapure water in turn to form a clear solution under continuous stirring for $10 \mathrm{~min}$. Afterwards, a piece of treated NF $(1 \mathrm{~cm} \mathrm{x} 4 \mathrm{~cm})$ and the above solution were transferred into a $50 \mathrm{~mL}$ stainlesssteel Teflon-lined autoclave and heat-treated at $120^{\circ} \mathrm{C}$ for $12 \mathrm{~h}$. After that, the as-obtained P-NiCoV-LTH/NF was collected and rinsed with distilled water and ethanol, and then dried at $60{ }^{\circ} \mathrm{C}$ for $24 \mathrm{~h}$ under a vacuum condition. The $\mathrm{Co} / \mathrm{V}$ molar ratio of $\mathrm{P}-\mathrm{NiCoV}-\mathrm{LTH} / \mathrm{NF}$ is $1: 1$. In addition, different molar ratios of $\mathrm{Co} / \mathrm{V}$ solution (3:1 and 5:1 for the synthesis of M-NiCoV-LTH/NF and S-NiCoV- 
LTH/NF at the same hydrothermal conditions, respectively) was obtained by mixing $\mathrm{CoCl}_{2} \cdot 6 \mathrm{H}_{2} \mathrm{O}$ and $\mathrm{VCl}_{3}$ in $30 \mathrm{ml} \mathrm{H} \mathrm{H}_{2} \mathrm{O}$, while the total amount of metal ions $(\mathrm{Co} / \mathrm{V})$ was kept to $3.6 \mathrm{mmol}$.

\section{Characterizations}

The morphology and structure of as-prepared samples were characterized by field emission scanning electron microscope (FESEM, Hitachi, S-4800) and transmission electron microscope (TEM, HRTEM, FEI Tecnai G2 F20S-TWIN) at an accelerating voltage of $200 \mathrm{kV}$. The crystal structures were determined by a Rigaku, D/max-2200pc X-ray diffractometer with $\mathrm{Cu} \mathrm{K} \alpha(\lambda=0.15406 \mathrm{~nm})$ at a scanning rate of $4 \% \mathrm{~min}$. The surface elemental compositions and valence states of the samples were collected with X-ray photoelectron spectrometer (XPS, AXIS SUPRA with monochromatic $\mathrm{Al} \mathrm{K}_{\alpha}$ radiation as the X-ray source).

\section{Electrochemical measurements}

All electrochemical tests were carried on an electrochemical workstation (CHI 660E, Shanghai Chen Hua) in a three-electrode system with an $\mathrm{Hg} / \mathrm{HgO}$ electrode and a graphite rod were used as the reference electrode and the counter electrode, respectively. The obtained samples with an area of $0.09 \mathrm{~cm}^{2}$ were directly evaluated as working electrodes to characterize catalytic performance for OER. Linear sweep voltammetry (LSV) curves were recorded at a scanning rate of $5 \mathrm{mV} \mathrm{s}^{-1}$ with $90 \%$ iR compensation. Tafel slopes was derived from OER polarization curve obtained at $5 \mathrm{mV} \mathrm{s}^{-1}$. The electrochemical surface areas 
(ECSA) were measured using $\mathrm{CV}$ technique with a potential range between 0.2 and $0.3 \mathrm{~V}$ under different scan rates ranging from 20 to $120 \mathrm{mV} \mathrm{s}^{-1}$. The doublelayer capacitance $\left(\mathrm{C}_{\mathrm{dl}}\right)$ was determined by taking cyclic voltammetry and the half of the slope was taken as the $C_{\mathrm{dl}}$ of the electrocatalyst. Electrochemical impedance spectroscopy (EIS) was carried in the frequency range from $0.1 \mathrm{~Hz}$ to $100 \mathrm{kHz}$. The long-term stability test was evaluated with amperometric i-t curve measurement. All potentials were referenced to the reversible hydrogen electrode (RHE). All the electrochemical measurements were recorded with the iR compensation at ambient temperature. Before each test, the electrolyte was bubbled with $\mathrm{O}_{2}$ to eliminate dissolved oxygen in the solution.

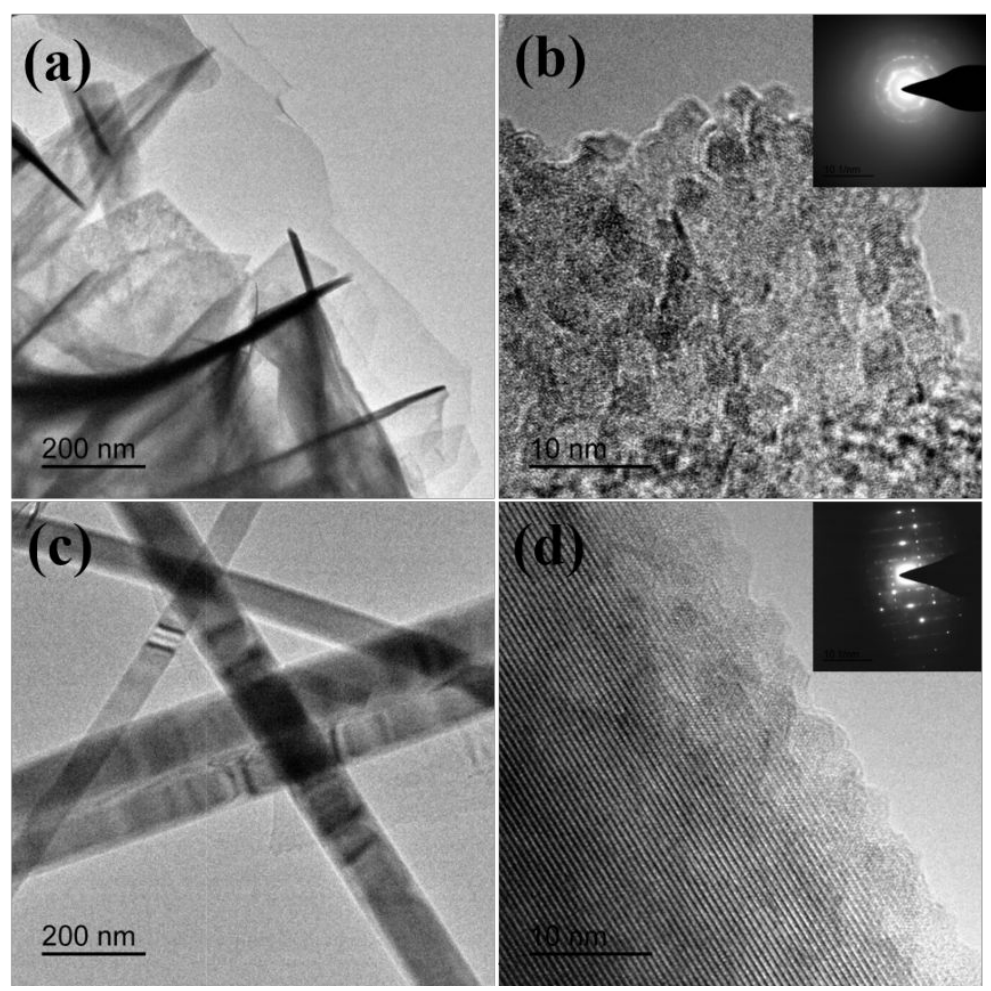


Figure S1 The TEM image of (a)(c) M-NiCoV-LTH/NF and (b)(d) HRTEM image of MNiCoV-LTH/NF.
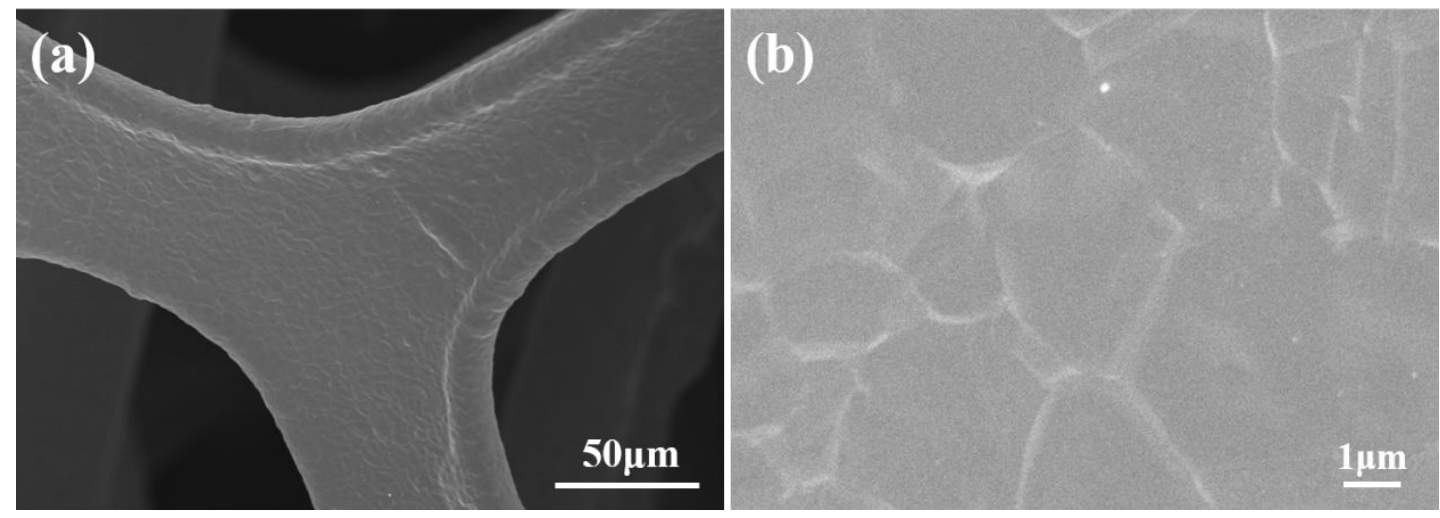

FigureS2 (a), (b) SEM images of pure NF.
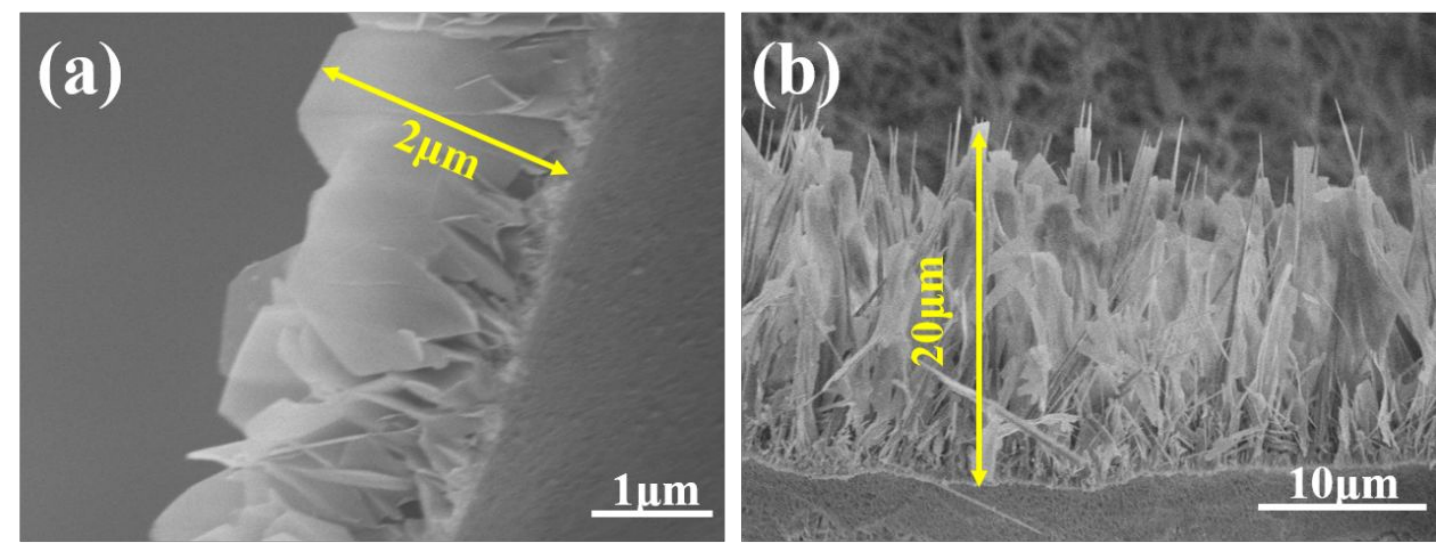

Figure S3 The SEM images of (a) P-NiCoV-LTH/NF and (b) S-NiCoV-LTH/NF

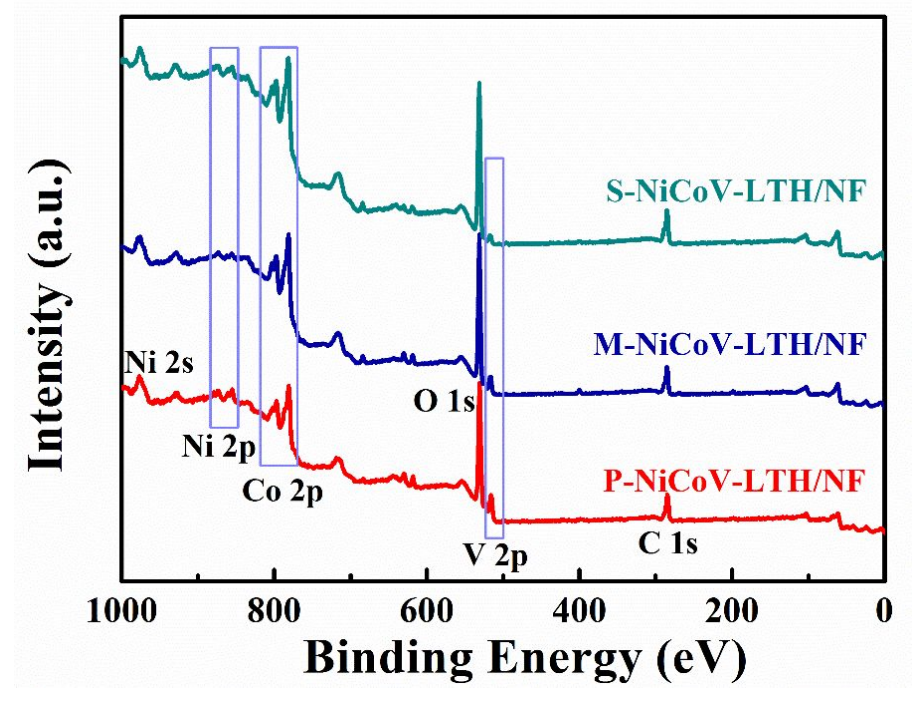


Figure S4 The survey spectra of the three samples (P-NiCoV-LTH/NF, M-NiCoVLTH/NF and S-NiCoV-LTH/NF).

\begin{tabular}{|c|c|c|c|}
\hline Catalysts & P-NiCoV-LTH/NF & M-NiCoV-LTH/NF & S-NiCoV-LTH/NF \\
\hline Element & $1.03 \%$ & $0.41 \%$ & $0.11 \%$ \\
\hline V & $2.65 \%$ & $1.67 \%$ & $0.76 \%$ \\
\hline Co & $6.77 \%$ & $7.8 \%$ & $8.25 \%$ \\
\hline
\end{tabular}

Figure S5 The atomic percentage of the three samples (P-NiCoV-LTH/NF, M-NiCoVLTH/NF and S-NiCoV-LTH/NF).

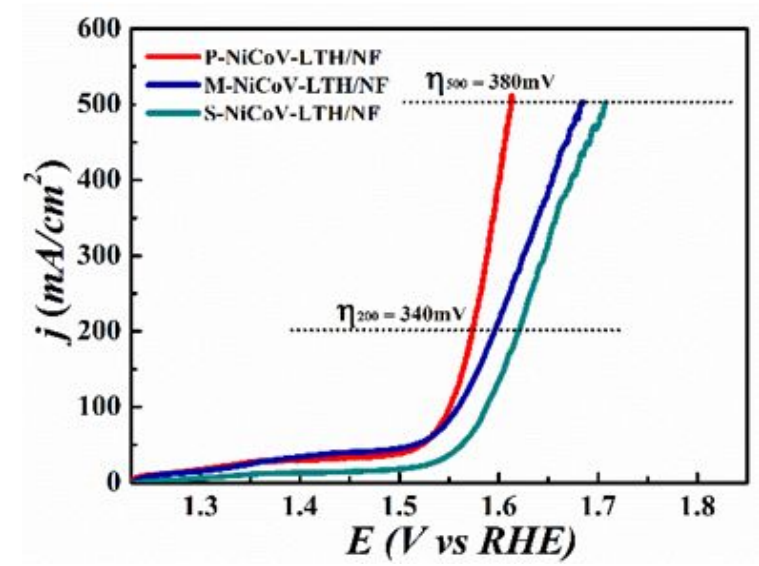

Figure S6 OER polarization curves of P-NiCoV-LTH/NF, M-NiCoV-LTH/NF and S$\mathrm{NiCoV}-\mathrm{LTH} / \mathrm{NF}$ in $1.0 \mathrm{M} \mathrm{KOH}$ at a scan rate of $5.0 \mathrm{mV} \mathrm{s}^{-1}$ (the electrode area: $0.8 \mathrm{~cm}^{2}$ ).
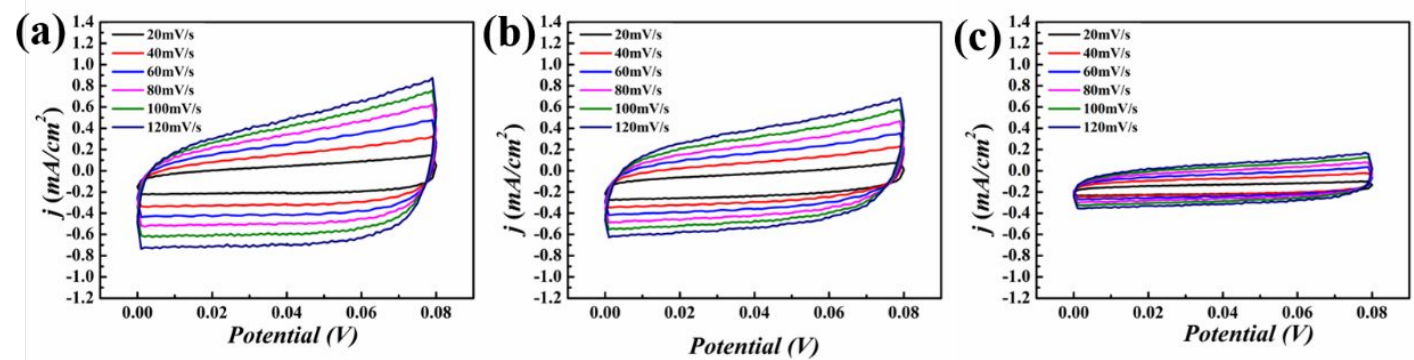

Figure S7 CV curves of (a) P-NiCoV-LTH/NF and; (b) M-NiCoV-LTH/NF and (c) S$\mathrm{NiCoV-LTH/NF}$ at different scan rates. 


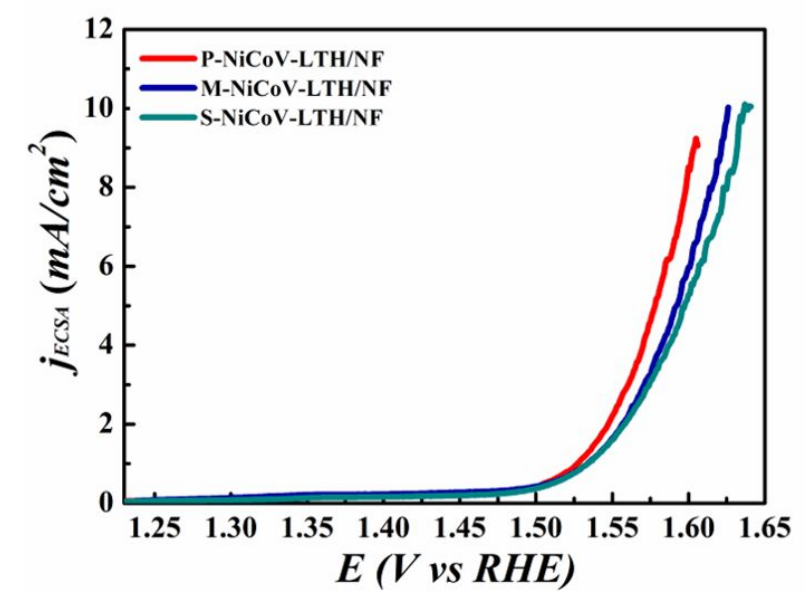

Figure S8 OER ECSA-normalized LSV curves of P-NiCoV-LTH/NF, M-NiCoVLTH/NF and S-NiCoV-LTH/NF.

The current densities measured have been normalized by the electrochemically active surface area $(E C S A)$ value $\left(j_{E C S A}=\frac{j}{E C S A}\right)$. As shown in Figure S8, the P-NiCoVLTH/NF still exhibits the best catalytic activity, implying the improved intrinsic OER activity. ${ }^{1}$

The ECSA of a catalyst sample is calculated from the double-layer capacitance according to the following equation:

$$
\operatorname{ECSA}=\frac{C_{d l}}{C_{S}}
$$

Cs presents the specific capacitance, the value of which is $0.040 \mathrm{mF} \mathrm{cm}^{-2}$ in $1 \mathrm{M}$ $\mathrm{KOH}$ based on the previous reported literature. ${ }^{2}$ 

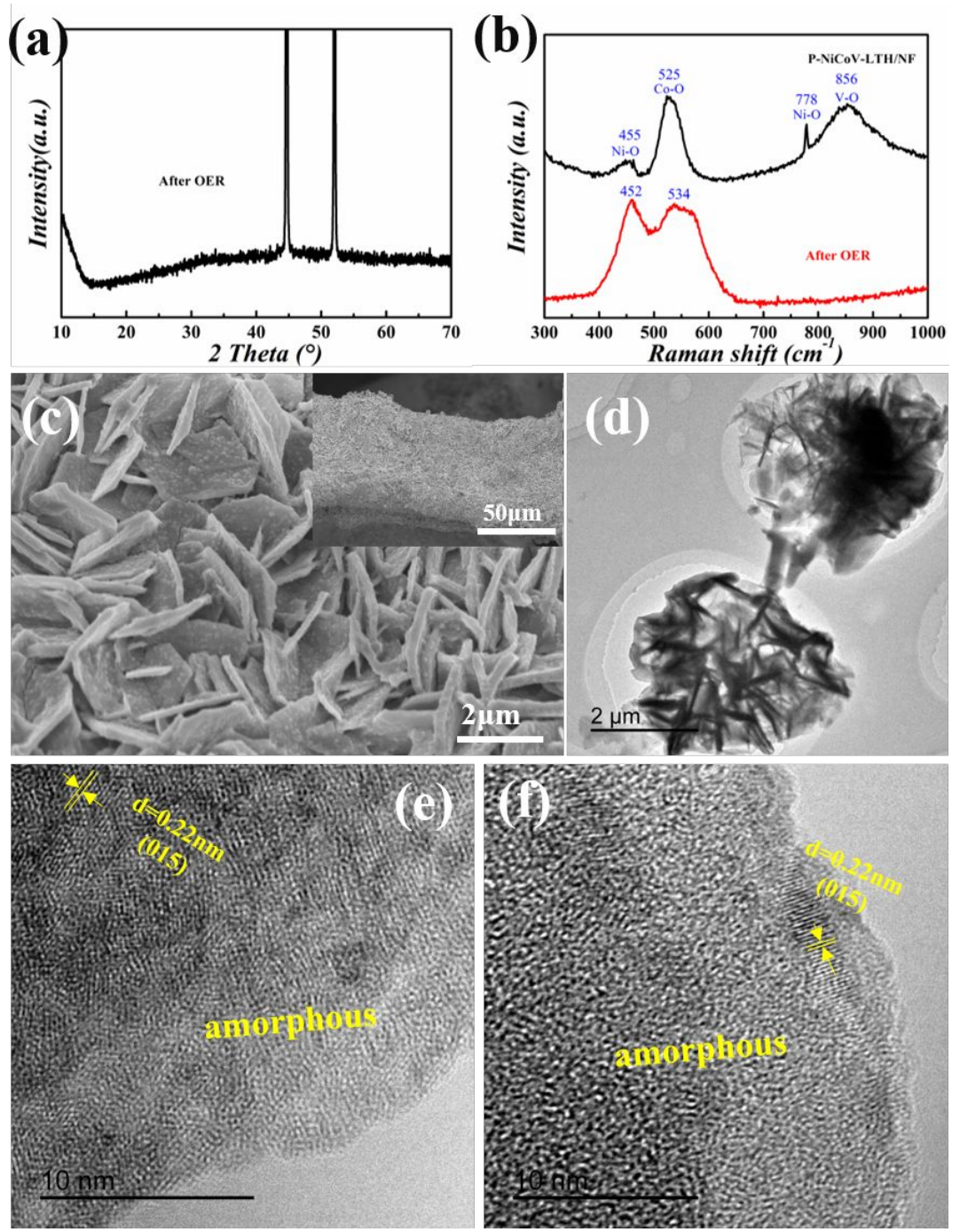

Figure S9 (a)XRD pattern; (b)Raman spectra; (c)SEM images; (d)TEM image; (e, f) HRTEM images of P-NiCoV-LTH/NF after OER test.

XRD, SEM images, TEM images and Raman spectra of P-NiCoV-LTH/NF after OER test have been provided. As shown in Figure S9(a), only the two typical diffraction peaks of $44.5^{\circ}$ and $51.8^{\circ}$ corresponding to the NF substrate were found while other peaks disappeared, which can be speculated to be amorphous phase generated during OER. As shown in Figure S9(b), P-NiCoV-LTH/NF possesses the four bands at 455, 525,778 and $856 \mathrm{~cm}^{-1}$, in which two bands at 455 and $778 \mathrm{~cm}^{-1}$ are attributed to the stretching $\mathrm{Ni}-\mathrm{O}(\mathrm{H})$ bond, one band at $525 \mathrm{~cm}^{-1}$ is assigned to the Co-O symmetric stretching mode of NiCo-LDH ${ }^{3,4}$ and the obvious peak located at $856 \mathrm{~cm}^{-1}$ can be attributable to the V-O vibrations. ${ }^{5}$ However, in the Raman spectrum of P-NiCoVLTH/NF after OER, the characteristic peaks in the V-O band disappeared and there are only two distinct bands of $452(\mathrm{Ni}-\mathrm{O})$ and $534(\mathrm{Co}-\mathrm{O}) \mathrm{cm}^{-1}$. This phenomenon may be 
due to the strong affinity of the $\mathrm{V}$ species to oxygen atoms, and the leakage of $\mathrm{V}$ occurred during the OER electrocatalysis process. ${ }^{6,7}$ The SEM and TEM images of PNiCoV-LTH/NF after OER test has been provided in Figure S9 (c, d). It is found that the nanosheets structure of the $\mathrm{P}-\mathrm{NiCoV}-\mathrm{LTH} / \mathrm{NF}$ were well-maintained, proving the excellent structural stability of the samples. Moreover, the HRTEM images (Figure S9 $(e, f))$ show a large number of amorphous structures and a small number of the lattice fringe with interplanar distances of $0.22 \mathrm{~nm}$, which match well with the (015) planes of LTH. The amorphous structures can be speculated as Ni/Co oxyhydroxides, which are often reported as real active substances in OER processes. This result is matched with $\mathrm{XRD}$ and Raman results. The all results from above characterization suggested the good stability of P-NiCoV-LTH/NF for the long-time OER test.

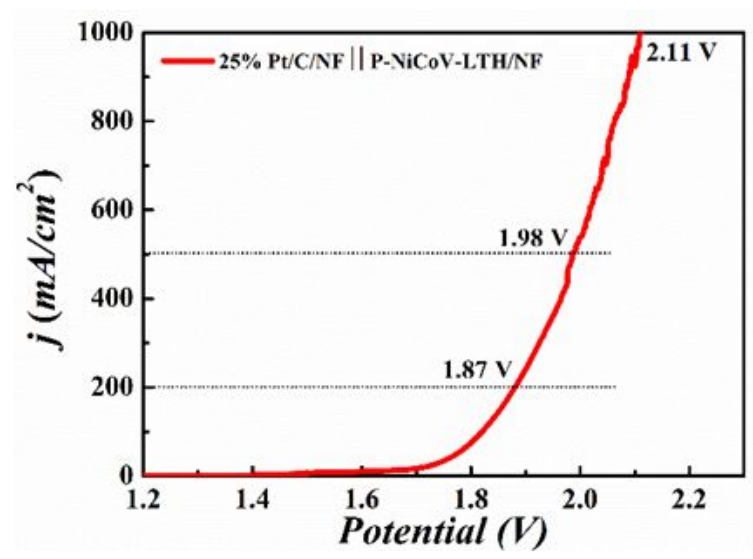

Figure S10 Overall water-splitting performance of P-NiCoV-LTH/NF || $25 \% \mathrm{Pt} / \mathrm{C} / \mathrm{NF}$ with a scan rate $5 \mathrm{mV} \mathrm{s}^{-1}$ in $1 \mathrm{M} \mathrm{KOH}$. 
Table S1. Comparison of the electrocatalytic performance of P-NiCoV-LTH/NF with other OER catalysts reported recently in $1 \mathrm{M} \mathrm{KOH}$ solution

\begin{tabular}{|c|c|c|c|c|c|c|}
\hline Catalyst & $\begin{array}{c}\text { Current } \\
\text { density } \\
\left(\mathrm{mA} / \mathrm{cm}^{2}\right)\end{array}$ & $\begin{array}{l}\text { Overpotential } \\
\text { at the } \\
\text { Corresponding } \\
j(\mathrm{mV})\end{array}$ & $\begin{array}{c}\text { Stability } \\
\text { (h) }\end{array}$ & $\begin{array}{c}\text { mass } \\
\text { loading } \\
\left(\mathrm{mg} \mathrm{cm}^{-2}\right)\end{array}$ & $\begin{array}{l}\text { thickness } \\
\text { of } \\
\text { substrate }\end{array}$ & Reference \\
\hline P-NiCoV-LTH/NF & $\begin{array}{c}100 \\
200 \\
500 \\
1000\end{array}$ & $\begin{array}{l}285 \\
310 \\
340 \\
373\end{array}$ & 180 & 2.75 & $1.5 \mathrm{~mm}$ & This work \\
\hline $\mathrm{NiFe}$ LDH/NF & 10 & 269 & 6000 & I & $1.5 \mathrm{~mm}$ & $\begin{array}{c}\text { Nat. Commun., } \\
\text { 2018, } 2609\end{array}$ \\
\hline $\begin{array}{c}\text { NiFeLDH- } \\
\text { NS@DG10/NF }\end{array}$ & 10 & 210 & 10 & 0.28 & l & $\begin{array}{c}\text { Adv. Mater. 2017, } \\
\text { 29, } 1700017 .\end{array}$ \\
\hline NiFe LDH/NF & $\begin{array}{c}10 \\
100\end{array}$ & $\begin{array}{l}219 \\
245\end{array}$ & 240 & l & l & $\begin{array}{c}\text { J. Mater. Chem. A, } \\
\text { 2019,7, } 22889\end{array}$ \\
\hline NiFe LDH/NF & $\begin{array}{c}10 \\
100\end{array}$ & $\begin{array}{l}180 \\
222\end{array}$ & 20 & 0.51 & I & $\begin{array}{l}\text { Sci. China Mater. } \\
\text { 2019, 62, 681-689 }\end{array}$ \\
\hline NiCo-LDH/NF & $\begin{array}{c}10 \\
180\end{array}$ & $\begin{array}{l}271 \\
470\end{array}$ & 20 & 1 & I & $\begin{array}{c}\text { Dalton Trans., } \\
\text { 2017,46, 8372-8376 }\end{array}$ \\
\hline NiCo LDH/CP & $\begin{array}{c}10 \\
300\end{array}$ & $\begin{array}{l}367 \\
683\end{array}$ & 6 & I & I & $\begin{array}{c}\text { Nano Lett. 2015, 15, } \\
1421 .\end{array}$ \\
\hline NiFe-LDH-20s/NF & $\begin{array}{c}10 \\
100 \\
200\end{array}$ & $\begin{array}{l}240 \\
306 \\
338\end{array}$ & 15 & I & l & $\begin{array}{l}\text { J. Power Sources } \\
\text { 2017, 365, 320-326 }\end{array}$ \\
\hline $\begin{array}{c}\mathrm{NiFe}_{2} \mathrm{O}_{4} / \mathrm{NiFe} \\
\mathrm{LDH} / \mathrm{NF}\end{array}$ & 100 & 213 & 20 & 2.8 & I & $\begin{array}{c}\text { ACS Appl. Mater. } \\
\text { Interfaces, 2018, 10, } \\
\text { 26283-26292 }\end{array}$ \\
\hline $\mathrm{NiFeCr}$ LDH/CP & 30 & 370 & 6 & 0.5 & $0.19 \mathrm{~mm}$ & $\begin{array}{c}\text { Adv. Energy Mater. } \\
2018,1703189\end{array}$ \\
\hline $\begin{array}{c}\mathrm{Ni}_{3} \mathrm{~S}_{2} @ \mathrm{NiV}- \\
\mathrm{LDH} / \mathrm{NF}\end{array}$ & 100 & 330 & 100 & 0.71 & $1.5 \mathrm{~mm}$ & $\begin{array}{c}\text { Nanoscale, 2019,11, } \\
\mathbf{8 8 5 5 - 8 8 6 3}\end{array}$ \\
\hline
\end{tabular}




\begin{tabular}{|c|c|c|c|c|c|c|}
\hline $\mathrm{Co}_{1} \mathrm{Mn}_{1} \mathrm{CH} / \mathrm{NF}$ & 100 & 340 & 18 & 5.6 & $1.8 \mathrm{~mm}$ & $\begin{array}{c}\text { J. Am. Chem. Soc. } \\
\text { 2017, 139, } \\
8320-8328\end{array}$ \\
\hline $\begin{array}{c}\text { NiFe LDH@ } \\
\text { NiCoP/NF }\end{array}$ & 100 & 350 & 100 & 2 & $1.6 \mathrm{~mm}$ & $\begin{array}{l}\text { Adv. Funct. Mater. } \\
\text { 2018, 28, } 1706847\end{array}$ \\
\hline NiFeV LDHs/NF & 100 & 233 & 18 & 2.8 & I & $\begin{array}{c}\text { Adv. Energy Mater. } \\
2018,1703341\end{array}$ \\
\hline $\begin{array}{l}\mathrm{NiFe}-\mathrm{LDH} / \\
\mathrm{NiCo}_{2} \mathrm{O}_{4} / \mathrm{NF}\end{array}$ & 100 & 390 & 10 & 4.9 & $1.6 \mathrm{~mm}$ & $\begin{array}{c}\text { Acs Appl. Mater. } \\
\text { Interfaces, 2017, 9, } \\
1488-1495\end{array}$ \\
\hline
\end{tabular}

\section{References:}

1. Niu, Y.; Li, W.; Wu, X.; Feng, B.; Yu, Y.; Hu, W.; Li, C. M. Amorphous nickel sulfide nanosheets with embedded vanadium oxide nanocrystals on nickel foam for efficient electrochemical water oxidation. J.Mater. Chem. A 2019, 7, 10534-10542.

2. C. L. McCrory, S. Jung, J. Peters, T. Jaramillo, Benchmarking Heterogeneous Electrocatalysts for the Oxygen Evolution Reaction, J. Am. Chem. Soc. 2013, 135, 16977-16987.

3. X Han, Y Niu, C Yu, Z. Liu, J. Qiu. Ultrafast Construction of Interfacial Sites by Wet Chemical Etching to Enhance Electrocatalytic Oxygen Evolution. Nano Energy, 2019, 69, 104367.

4. L. Huang, D. Chen, Y. Ding, S. Feng, Z. Wang, M. Liu. Nickel-Cobalt Hydroxide Nanosheets Coated on $\mathrm{NiCo}_{2} \mathrm{O}_{4}$ Nanowires Grown on Carbon Fiber Paper for High-Performance Pseudocapacitors. Nano Lett. 2013, 13, 3135-3139.

5. B. Tao, X Li, C Ye, Q. Zhang, Y. Deng, N. Li. One-step hydrothermal synthesis of cobalt-vanadium based nanocomposites as bifunctional catalysts for overall water splitting. Nanoscale 2019, 11, 18238-18245.

6. Liu, Q.; Huang, J.; Cao, L.; Kajiyoshi, K.; Feng, L., V-Doping Triggered Formation and Structural Evolution of Dendritic $\mathrm{Ni}_{3} \mathrm{~S}_{2} @ \mathrm{NiO}$ Core-Shell Nanoarrays for Accelerating Alkaline Water Splitting. Acs Sustain. Chem. Eng. 2020, $8,6222-6233$

7. Yang, D.; Cao, L.; Feng, L.; Huang, J.; Hai, G., Formation of hierarchical $\mathrm{Ni}_{3} \mathrm{~S}_{2}$ nanohorn arrays driven by insitu generation of $\mathrm{VS}_{4}$ nanocrystals for boosting alkaline water splitting. Appl. Catal. B: Environ. 2019, 257, 117911. 